\title{
Advocacy for persons with rare diseases: a strategy for creating influence
}

\author{
Christopher C Chang, Victoria Gall and Matthew H Liang
}

Approximately 7,000 rare diseases-operationally defined in the US as disorders that affect 200,000 people or fewer-exist (NORD Guide to Rare Disorders. Lippincott Williams \& Wilkins, Philadelphia, 2003). Of these disorders, more than 100 are rheumatic diseases (Decker JL [1983] Arthritis Rheum 26: 1029-1032). Over 800 organizations for rare disorders exist.

Rare diseases share many features. They are not covered well in medical school curricula. Most primary care physicians lack sufficient experience to prescribe and coordinate the diverse services needed over a patient's lifetime. Furthermore, most of these diseases are considered to appeal to 'small markets' and are neglected for research and development by the pharmaceutical and medical device industries.

To assess the organizations for rare diseases, we surveyed 115 groups randomly selected from the National Organization for Rare Disorders (NORD) Guide to Rare Disorders (five of which were related to rheumatology). A six-page questionnaire was developed by Ewan Goligher and Margaret Donlon in conjunction with key informants from the Lupus Foundation, the Marfan Association, and the Osteogenesis Imperfecta Foundation, and sent out up to three times. Overall, 46 (40\%) returned the survey; 20 (17\%) of the surveys were returned as 'addressee unknown'. Four independent reviewers classified open-ended responses.

The survey showed that funding was universally viewed as the greatest obstacle to meeting patient needs. Most organizations described patient, physician, and community education as their greatest priorities, and the provision of educational materials, research updates, and physician education as their most common service.

Surprisingly, there was little interaction between groups. The most common form $(80 \%)$ of communication was hyperlinks to other groups on the organization's website.
In aggregate, these groups could be a powerful voice to shape the design and reimbursement of health services, research, and social policy

C Chang is a Medical Student at New York University School of Medicine. $V$ Gall is a Senior Project

Manager in the Section of Clinical Sciences, Division of Rheumatology, Immunology and Allergy at the Brigham and Women's Hospital at Harvard Medical School in Boston, MA, USA, where MH Liang is a Professor of Medicine. MH Liang is also a member of the Nature Clinical Practice Rheumatology Advisory Board and Professor of Health Policy and Management at the Harvard School of Public Health, Boston, MA.

\section{Competing interests}

The authors declared no competing interests.

www.nature.com/clinicalpractice doi:10.1038/ncprheum0561
Some groups $(29 \%)$ have a common registry and others (24\%) share membership. Fewer (15\%) had collaborative fundraising efforts.

In the US, patients with rare diseases number 25 million people-almost $12 \%$ percent of the population. Independent advocacy by individual groups dilutes the potential political influence. In aggregate, these groups could be a powerful voice to shape the design and reimbursement of health services, research, and social policy.

The 1983 Orphan Drug Act, which guarantees the developer of an orphan product 7 years of market exclusivity after its approval by the FDA, is an example of a successful policy framed for all rare diseases. From 1983-2002, the FDA approved nearly 250 orphan products. Currently, nearly 800 products for rare diseases are under development. However, further collaborative lobbying is needed to lower the prohibitively high costs of specialized drugs in the absence of government subsidies.

An example of an inclusive research policy is the NIH Autoimmunity Centers of Excellence, a research network for rare rheumatic diseases. This organization provides resources, continuity, and economies of scale not possible by funding the study of individual diseases.

Influencing decisions regarding resource allocation involves creating consensus around the pertinent issues and getting the support (in terms of votes). Rare disease organizations are defined by their prevalence and, as such, have essentially been neglected, and their constituents' common interests marginalized. For issues that affect all patients with rare disease, such as universal health care, subsidies or payment for expensive new therapies, specialized equipment or appliances, and access to specialists throughout the course of the disease, advocacy as a unified group of 25 million individuals with rare conditions would be a powerful and, potentially more successful, voice. 